\title{
Correlation between the reading of a thermal imaging camera and the focus of an electronic component thermogram
}

by Krzysztof DZIARSKI

\author{
*Poznań Univ. of Technology, 60-965, Piotrowo 3A, Poznań, Poland, Krzysztof.Dziarski@put.poznan.pl
}

Precise thermal management of electronic components is a vital issue as an excessive temperature of an element may indicate a failure in its operation or over-generation of power within the element. The latter may be caused - for example - by incorrect determination of its operating point. In both cases the condition is undesirable. Furthermore, continued operation of an element with excessive temperature is likely to reduce its service life and compromise its performance. And there will be losses of power generated by the element. On the other hand, an inadequate temperature of an electronic element may be a sign of oversized cooling. Particularly in the case of power electronic components oversizing of the cooling system can be costly. One of the methods enabling assessment of the temperature of a semiconductor component involves thermovision measurement. It consists in using a thermal imaging camera to measure the temperature of the plastic surface over a semiconductor connection. Then, based on a unidimensional model, the semiconductor connection temperature can be determined. It is worth mentioning that in this case the temperature measured using a thermal imaging camera is the input quantity. The more accurate the temperature measurement of the plastic over the connection the more precise the determination of the semiconductor temperature. And the accuracy of the value detected using a thermal imaging camera depends on such factors as emissivity, reflected temperature, chemical composition of the air, vertical and horizontal observation angle, air humidity, and the distance between the camera lens and the target surface. In the course of work the author observed that yet another parameter has to be considered: the focus of the recorded thermogram. Changes in the reading of a thermal imaging camera depending on the selected focus of the thermogram result from the applied focusing method. The focus of the thermogram can be selected by changing the distance between the lens and the target surface or by adjusting the position of the lens using a focus adjustment ring. In both cases for only one setting the image is obtained in the detector plane. In other cases the image is outside the plane. If the image is not in obtained within the matrix plane the whole energy of the observed surface is not received by the detectors. The validity of the focus selection method with regard to the recorded thermogram was to be proved empirically. In order to perform an experimental verification a test rig was prepared. It comprises a chamber with a FLIR E50 thermal imaging camera fitted with an extra $2 x$ close-up lens, T197200. The focus ring on the camera lens had an adjustment range of 0-45 degrees with 1.5 degree steps, operated by a stepper motor controlled by a PLC located outside the chamber. The focus ring set point was selected by means of a touch panel of the PLC unit. Additionally, the panel displayed information about the current position of the lens ring which enabled adjustment without opening the chamber. Inside the chamber was a temperature and humidity sensor to monitor the test conditions. The inside of the chamber was lined with grey porous PU foam. Placing the camera in the chamber provided stable conditions for thermovision measurements as the ambient temperature stabilized. Moreover, enclosing the camera separated the object of observation from the source of optical radiation which could otherwise affect the results. The test object was a Pt1000 temperature sensor in an enclosure of $2.1 \times 2.3 \mathrm{~mm}$. The known surface temperature of the sensor was a benefit in the test. During the measurements the surface temperature of the sensor differed from the ambient temperature due to spontaneous heating - the current flowing through the sensor during the test exceeded the value of the current used for its operation. Using the know values of current and voltage the sensor resistance was determined and then its surface temperature. The obtained surface temperature value was compared to the value read by the thermal imaging camera. Prior to the measurements the values of all parameters according to the test conditions were entered using the camera control panel. The only adjustment was the position of the focus ring on the lens. Three series of thermograms were recorded for the set values of the ring angle. Next, the focus of each thermogram was expressed using one of the six focus measures: EOG, EOL, SML, Variance, Tenegrad and SF [1, 2, 3]. The obtained values of Focus measures for individual thermograms were standardized and the best measure was determined to be EOG. It was observed that the absolute error value for the camera readings changed with the focus of the thermograms (Fig. 1). The best thermogram focus was established for the ring angle of $13.5^{\circ}$. The testing distance between the sensor and the camera lens was $33 \mathrm{~mm}$. The absolute error of the camera was determined by changing the distance between the sensor and the lens. It was found that the best focus for the recorded thermograms was achieved at a distance of $33 \pm 4 \mathrm{~mm}$. In the case of the other distances the focus decreased. The absolute error of the camera readings was found to increase as the focus decreased (Fig. 2). 


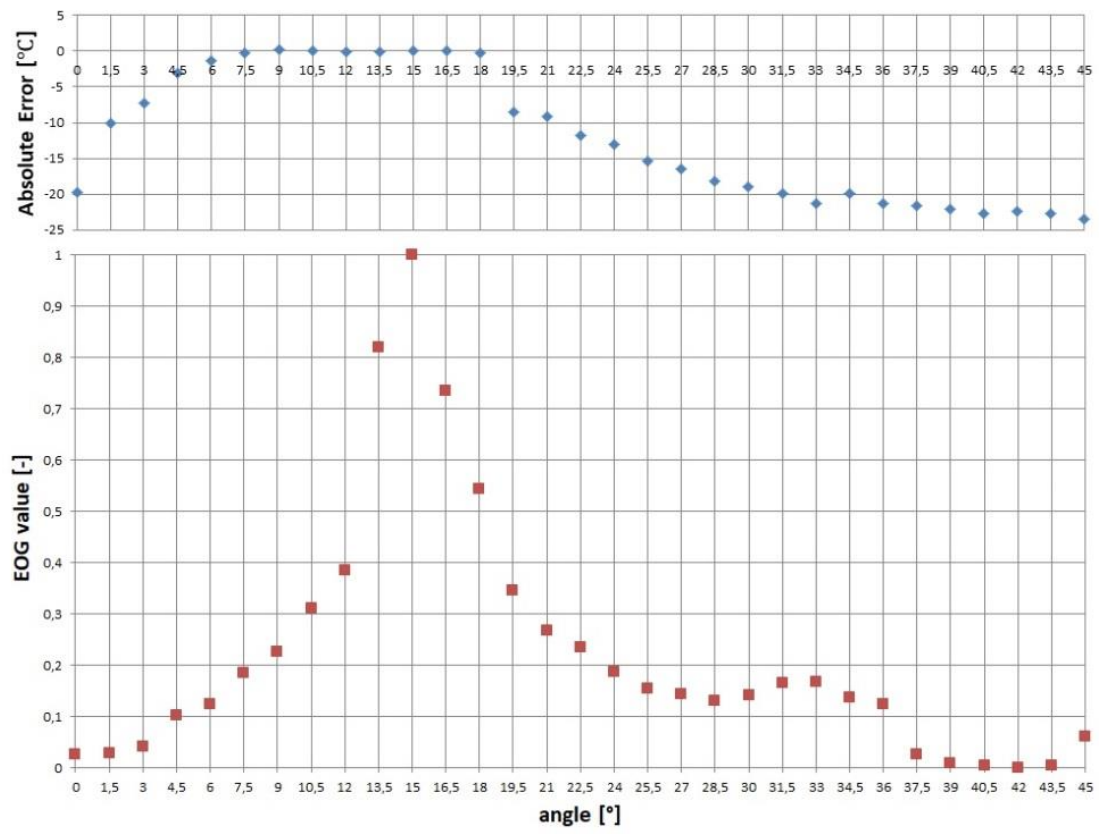

Fig. 1. Correlation between absolute error and focus ring angle, and between standardized EOG value and focus ring angle.

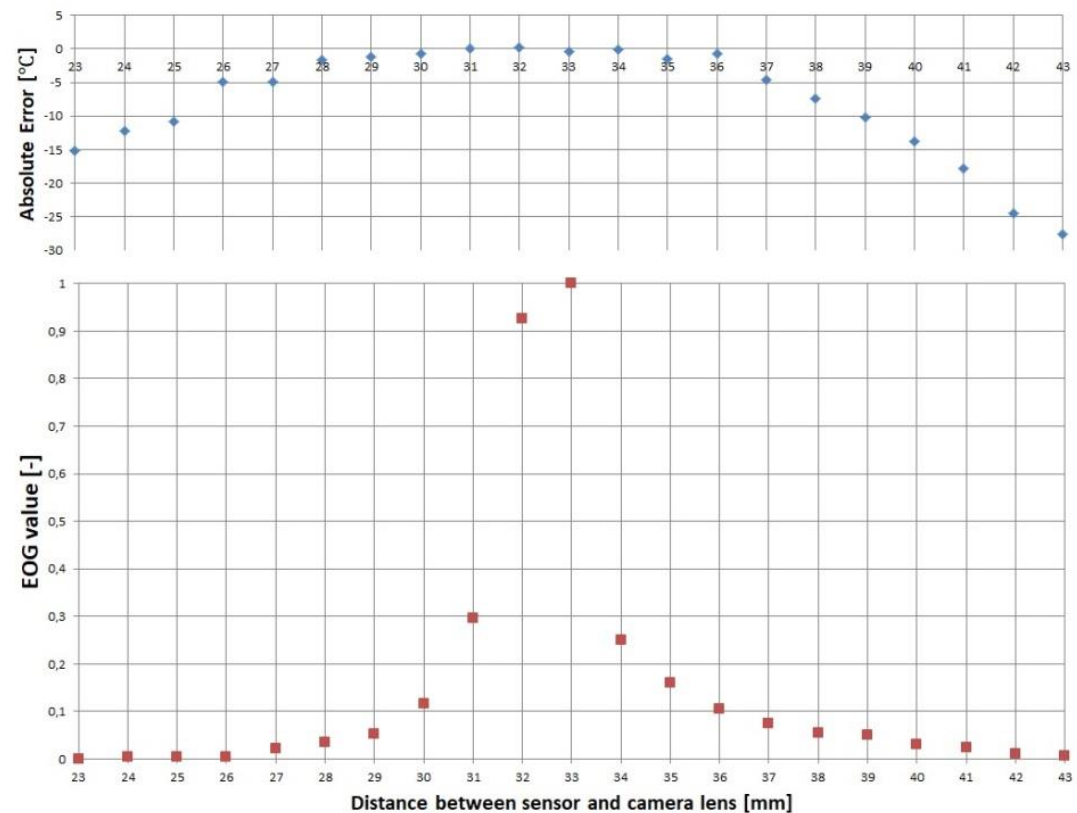

Fig. 2. Correlation between the sensor - lens distance and absolute error, and between standardize EOG value and the sensor - lens distance

\section{REFERENCES}

[1] H. Wei., J. Zhongliang, Evaluation of focus measures in multi-focus image Fusion, Pattern Recognition Letters, Volume 28, Issue 4, 1 March 2007, Pages 493-500.

[2] M. Faundez-Zanuy' ,J.Mekyska, V. Espinosa-Duró, On the focusing of thermal images, Pattern Recognition Letters, Volume 32, Issue 11, 1 August 2011, Pages 1548-1557

[3] X. Wang , Y. Wang A New Focus Measure for Fusion of Multi-Focus Noisy Images, 2010 International Conference on Computer, Mechatronics, Control and Electronic Engineering (CMCE). 